\title{
Gaze Control: A Developmental Perspective
}

\author{
Janette Atkinson and Oliver Braddick \\ Visual Development Unit, University College London, London, UK
}

\section{Introduction}

The chapter by Sandini et al. provides good examples of how the working of biological visual systems can help us to understand the design problems of an engineered system, and vice versa. However, it is important to bear in mind that the organization of a biological system has arisen through processes that are rather different from the design and manufacture of an engineered system. The biological system has to be understood as the end point of an evolutionary and a developmental process.

\section{Modularity and the evolution of the visual system}

At the present state of engineering, it does not appear a sensible objective to design a general purpose robot. Thus artificial vision systems usually have rather specific and limited goals. Furthermore, a modular approach makes their design a more tractable problem, so if there are different subgoals, they are treated fairly independently in the design process. Thus the present chapter treats gaze stabilization as a self-contained problem.

The modular approach to neural systems has also made them more tractable to understand, and the long evolutionary history of gaze stabilization encourages us to believe that there are special purpose mechanisms, which may have been conserved in evolution, to perform this task. However, these mechanisms have coevolved with other aspects of a system that achieves many diverse visual goals, such as guiding orienting actions and recognizing objects. The constraints and tradeoffs that might apply to the gaze stabilization problem in isolation do not necessarily apply when it is considered as part of this wider context. 


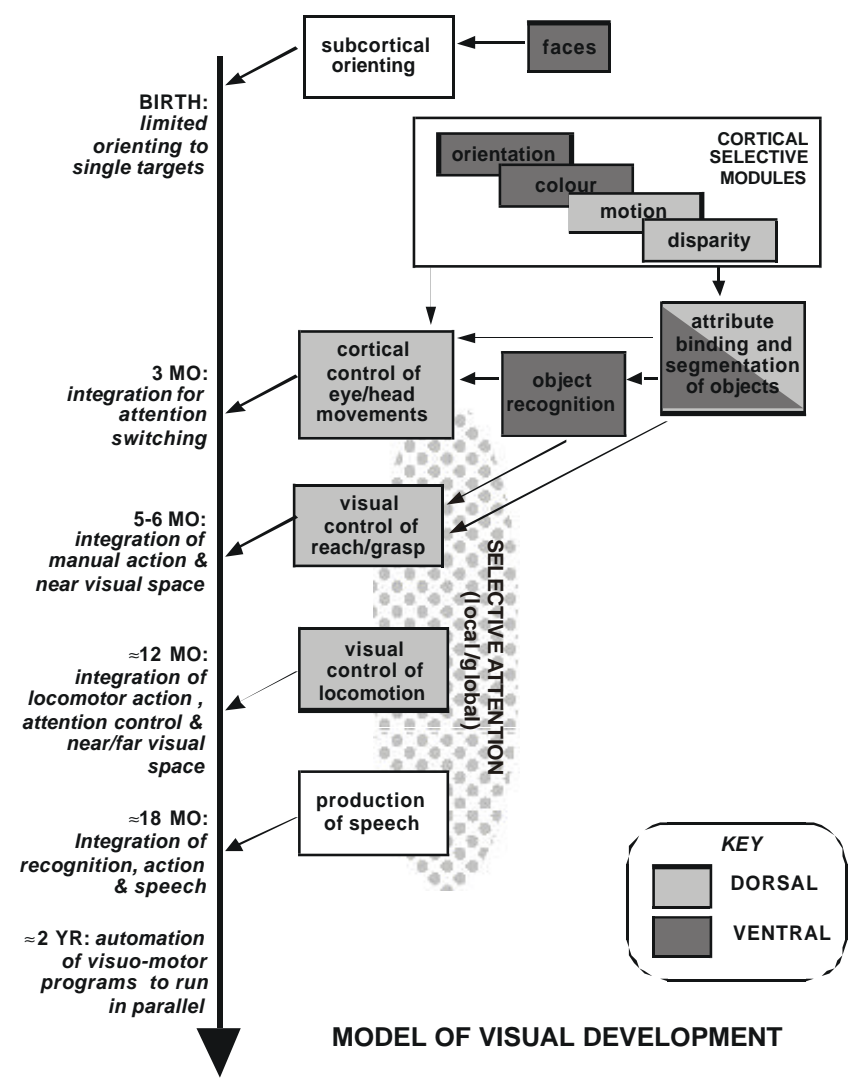

Fig. 1 Model of the developing visual system in human infants. The different integration processes, involving functioning of different action systems, are shown on the left hand side of the vertical line. Several different action systems become functional at different ages of development in the first two years of life. In temporal order of development these are (a) the newborn subcortically controlled orienting system of the head and eyes, involving reflexive eye movement systems; (b) cortical action systems controlling both saccadic and smooth pursuit eye movements, involving integration of subcortical and cortical (parietal, frontal) circuits. These circuits are also dependent on information from cortically selective modules for different visual attributes of objects, i.e. shape, colour, size, and those responsible for attribute binding and segmentation (parietal, temporal) which develop in the first months of life. (c) action modules for reaching and grasping in nearby space (parietal-frontal, temporal); (d) action modules for controlling locomotion, accompanied by mechanisms for attentional shifting between different scales of representation of space at different distances (parietal, frontal); (e) action modules for production of speech and integration of information from modules for object recognition, motor actions and speech; (f) action systems for automating visuo-motor programs and providing parallel processing across modules.

This problem is illustrated by the Sandini et al.'s discussion of "pre-attentive" processing. At several points they refer to target selection and attentional processes as being a "waste of resources" in gaze stabilization. The relative cost of 
different resources in computer engineering is not necessarily the same as that in the richly parallel, noisy environment of the brain. But in any case, if other functions of the visual system (e.g. the initiation of saccades) depend strongly on selective processes, then the resources are being used anyway. Thus selective processes might play a role in human gaze stabilization even if they did not make "economic" sense for achieving the goal of stabilization on its own. Work on single units in primates (e.g. Desimone and Duncan 1995; Desimone 1998; Seidemann and Newsome 1999), and human brain imaging studies (e.g. Tootell et al. 1998; Gandhi et al. 1999; Martinez et al. 1999) have shown that effects of selective attention are remarkably pervasive in visual areas of the cortex. It is likely that this information feeds into gaze stabilization mechanisms, although it may be possible to dissociate it from more basic computations underlying stability by exploiting the differential latency of different effects, as Sandini et al. suggest. This is an empirical question, which cannot be prejudged in terms of the notional resource demands of neural processing.

\section{The developmental context}

A second important aspect of biological vision is that its capabilities are achieved through a developmental process. At intermediate stages of development, different aspects of the system (e.g. acuity, binocularity, motion integration) are nearer or further from their mature state. The developing system therefore has to function with a different relation between its components from that we see in the mature system.

\section{Development of "space variant geometry"}

One example of this is the role of what the engineers call "space variant sensing" - in biological terms, the inhomogeneity of the retina. The mature human visual system contains much denser retinal sampling at the fovea than in surrounding regions, like Sandini et al.'s "log-polar" tessellation. However, in infancy the human fovea is poorly differentiated in terms of receptor density, and photoreceptors migrate considerably across the visual field in the course of development to achieve the final foveal packing (Youdelis and Hendrickson 1986). Thus, the kind of mechanism proposed by Sandini et al., which weights central vision because of its higher sampling density, would not achieve the right result in a human infant. We do not know whether this difference has any effects on the operation of gaze stabilization mechanisms in infancy. We do know, however, that even newborn infants' fixation behaviour operates to crudely bring significant targets (to which we assume the infant is attending) to the fovea, even though that fovea does not yet have the anatomical specialization that gives it higher resolu- 
tion than surrounding areas. This illustrates that the space-variant organization of human vision does not operate solely through differences in pixel density. Presumably the fovea-centred system is already embodied in cortical topography, and this is linked to oculomotor control at a very early stage of development.

The immaturity of the receptor distribution, coupled with oculomotor systems that are adapted to the not-yet-developed specialization of the fovea, would be expected to lead to a different functional balance from the mature state. This implies that the role of space-variant organization, suggested by Sandini et al., might lead to some interesting developmental predictions.

The orienting mechanism, bringing an object of interest onto the fovea for stable viewing by means of head and eye movements, which has been referred to above, is the first system in a series of visual action mechanisms developing in human infancy. A schematic diagram of this developmental sequence of action systems, including stabilizing systems, and its timescale is shown in figure 1 (after Atkinson, 2000).

Each of these action streams are likely to involve the integration of many cortical and subcortical areas. A scheme of the major action systems, which we have proposed, based on studies of non-human primates and neurological studies of adult patients (e.g. Jeannerod 1988; Milner and Goodale 1995; Rizzolatti et al. 1997), is shown in figure 2 (after Atkinson, 2000).

Here we see both separate and overlapping subcortical-cortical circuitry for saccadic eye movements and smooth pursuit movements, but there is also overlap in the adult systems with action modules for reaching and grasping.

\section{Developmental action systems}

The newborn orienting system and its relation to the model of Sandini et al. has already been briefly discussed. In the newborn infant this system functions suboptimally initially, with hypotonic saccades being made to fixate an initially peripheral target of interest (Aslin and Salapatek 1975). However, there is some debate as to just how hypotonic these saccades are when the infant is free to move both the head and eyes together to orient, rather than when the head is held in a fixed position, as in many studies.

Differences have also been found between one month and three months olds in the extent and accuracy of smooth pursuit, which implies at least in human development that there are different stabilizing mechanisms for providing information to enable targets to be smoothly tracked to those for saccadic movements in orienting. Many of the most detailed developmental studies of these stabilizing mechanisms in infants have been made by Claus von Hofsten and his colleagues (von Hofsten and Rosander 1996, 1997). They recorded the presence of smooth pursuit eye movements even in newborns, if targets of sufficient size and contrast were used and their velocity was kept relatively slow. They have also looked at 
the development of the initial coupling of eye and head movements, as the infants develop these stabilizing mechanisms. Fairly accurate coupling and a mature vestibular ocular response is achieved in the first few months of life, although many of the tracking eye movements observed in everyday situations in this period are saccadic, rather than continuous smooth pursuit. An understanding of the limitations and parameters in the developmental context should make it possible to extend and test the models of Sandini et al. for gaze stabilization in the human infant.

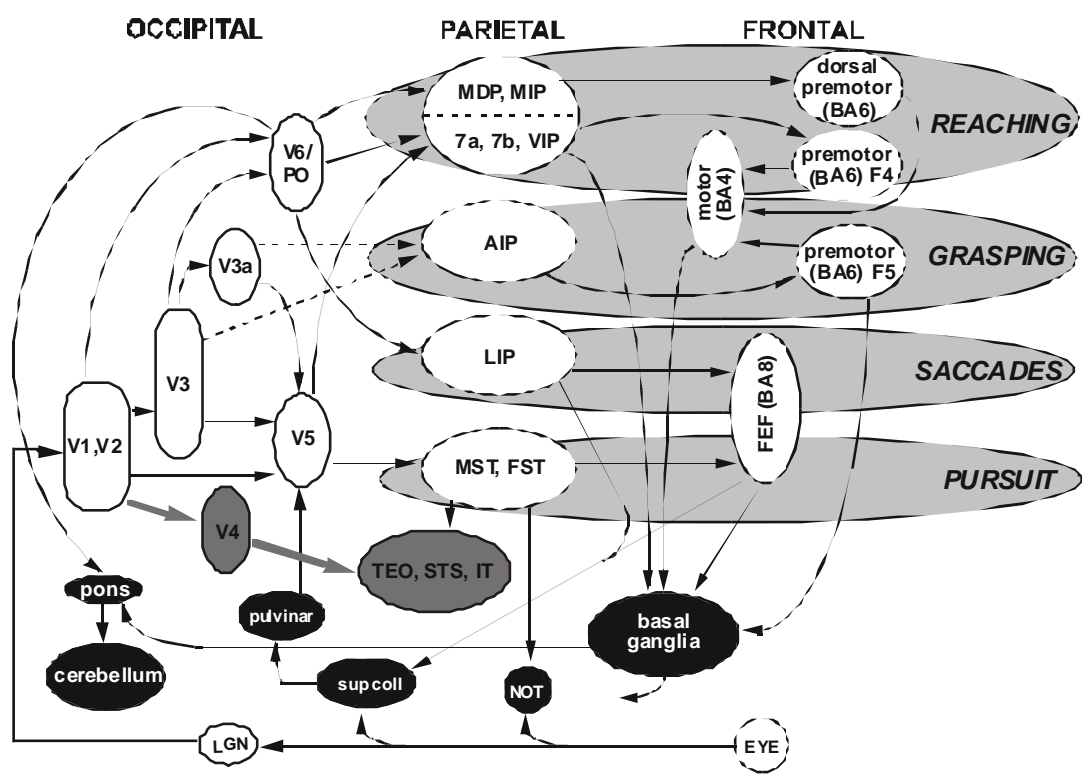

Fig.2 A schematic account of the areas contributing to different visuo-motor action streams in the primate brain (based on neurophysiological and neuropsychological findings). Four action streams are outlined; these are for reaching, grasping, saccadic eye movements and pursuit eye movements. BA: Brodmann's area; V1, V2, etc.: visual area 1, visual area 2, etc.; F4, F5: frontal areas; LIP: lateral intraparietal; V5 = MT: middle temporal, V6 = PO: parietal occipital; AIP: anterior intraparietal; MIP: medial intraparietal; MDP: mediodorsal parietal; VIP: ventral intraparietal; MST: medial superior temporal; FEF: frontal eye fields; TEO: posterior inferior temporal; IT: inferior temporal; STS: superior temporal sulcus; NOT: nucleus of the optic tract; sup coll: superior colliculus. (Reference source mainly: Rizzolatti et al. 1988; Milner and Goodale 1995; Jeannerod 1997).

\section{Binocularity and stabilization}

Sandini et al. give a very important role to binocular interactions, especially in maintaining stabilization against translational movements. As we can see from the schematic developmental model of figure 1, in human development binocular 
interaction is not one of the earliest features of cortical organization; systems sensitive to binocular disparity start to be come functional around three to four months of age (Birch 1993; Braddick 1996). Optokinetic and vestibulo-ocular reflexes for gaze stability certainly operate well before this age, as we have discussed above. However, their organization is different from the mature system. Up to three months, monocular OKN can be driven only by the temporal-to-nasal direction of movement for each eye (Atkinson and Braddick 1981). This has been attributed to the operation of a direct pathway from the retina to the contralateral midbrain nucleus of the optic tract (NOT) since the left NOT responds only to stimulus movement in the leftward direction and the right NOT only to rightward movement (Hoffmann and Schoppmann 1975; Hoffmann 1981). Nasal-to-temporal OKN appears to depend on development of a descending pathway to the NOT from binocular cells in cortex, including area MT/MST (Ilg and Hoffman 1993). Gross disruption or absence of the cortical input to NOT, e.g. in infants who have had early hemispherectomy to alleviate seizures due to developmental cortical malformations, leads to a loss of even the temporal-to-nasal response (Braddick et al. 1992; Morrone et al. 1999). However, even if the cortex on both sides is intact, the development of symmetrical OKN is disrupted by developmental problems of binocularity, in particular esotropic strabismus. It is not yet known whether this is because only binocular neurones can carry information to the NOT from the ipsilateral eye, or whether binocularity as such is important for normal optokinetic function. It is possible that the binocular pathway, as well as providing the basis for a symmetrical monocular OKN response, also subserves the modulation of optokinetic responses by binocular disparity, which Sandini et al. describe as providing stabilization against translational movements.

The approach through computational design issues, taken by Sandini et al. can therefore give insight into states of the developing system as well as the mature system. However, the developmental perspective also provides important tests of its relevance to the human brain.

\section{References}

Aslin RN, Salapatek P (1975) Saccadic localization of targets by the very young human infant. Percept Psychophys 17: 293-302

Atkinson J, Braddick OJ (1981) Development of optokinetic nystagmus in infants: an indicator of cortical binocularity? In: Fisher DF, Monty RA, Senders JW (eds) Eye movements: cognition and visual perception. Lawrence Erlbaum Associates, Hillsdale, N J, pp53-66

Atkinson J (2000) The developing visual brain. Oxford University Press, Oxford

Birch E (1993) Stereopsis in infants and its developmental relation to visual acuity. In: Simons K (ed) Early visual development: normal and abnormal. Oxford University Press, New York, pp 224-236

Braddick O, Atkinson J, Hood B, Harkness W, Jackson G, Vargha-Khadem F (1992) Possible blindsight in babies lacking one cerebral hemisphere. Nature 360: 461-463

Braddick O (1996) Binocularity in infancy. Eye 10: 182-188 
Desimone R (1998) Visual attention mediated by biased competition in extrastriate visual cortex. Phil Trans Roy Soc B 353: 1245-1255

Desimone R, Duncan J (1995) Neural mechanisms of selective visual attention. Ann Rev Neurosci 18: 193-222

Gandhi SP, Heeger DJ, Boynton GM (1999) Spatial attention affects brain activity in human primary visual cortex. Proc Natl Acad Sci USA 96: 3314-3319

Hoffmann K-P (1981) Neuronal responses related to optokinetic nystagmus in the cat's nucleus of the optic tract. In: Fuchs A, Becker W (eds) Progress in oculomotor research. Elsevier, New York, pp 443-454

Hoffmann K-P, Schoppmann A (1975) Retinal input to the direction sensitive cells of the nucleus tractus opticus of the cat. Brain Res 99: 359-366

von Hofsten C, Rosander K (1996) The development of gaze control and predictive tracking in young infants. Vision Res 36: 81-96

von Hofsten C, Rosander K (1997) Development of smooth pursuit tracking in young infants. Vision Res 37: 1799-1810

Ilg UJ, Hoffmann K-P (1993) Functional grouping of the cortico-pretectal projection. J Neurophysiol 70: 867-869

Jeannerod M (1988) The neural and behavioural organization of goal directed movements Oxford University Press, Oxford

Jeannerod M (1997) The cognitive neuroscience of action. Blackwell, Oxford

Martinez A, Anllo-Vento L, Sereno MI, Frank LR, Buxton RB, Dubowitz DJ, Wong EC, Hinrichs H, Heinze HJ, Hillyard SA (1999) Involvement of striate and extrastriate visual cortical areas in spatial attention. Nature Neurosci 2: 364-369

Milner AD, Goodale MA (1995) The visual brain in action. Oxford University Press, Oxford

Morrone MC, Atkinson J, Cioni G, Braddick OJ, Fiorentini A (1999) Developmental changes in optokinetic mechanisms in the absence of unilateral cortical control. NeuroReport 10: 2723-2729

Rizzolatti G, Fogassi L, Gallese V (1997) Parietal cortex: from sight to action. Current Opinion Neurobiol 7: 562-567

Rizzolatti G, Camarda R, Fogassi L, Gentilucci M, Luppino G, Matelli M (1988) Functional organization of area 6 in the macaque monkey II. Area F5 and the control of distal movements. Exp Brain Res 71: 491-507

Seidemann E, Newsome WT (1999) Effect of spatial attention on the responses of area MT neurons. J Neurophysiol 81: 1783-1794

Tootell RB, Hadjikhani N, Hall EK, Marrett S, Vanduffel W, Vaughan JT, Dale AM (1998) The retinotopy of visual spatial attention. Neuron 21: 1409-1422

Youdelis C, Hendrickson A (1986) A qualitative and quantitative analysis of the human fovea during development. Vision Res 26: 847-855 